\title{
Effects of stellar flybys on planetary systems: 3D modeling of the circumstellar disk's damping effects
}

\author{
G. Picogna and F. Marzari
}

\author{
Dipartimento di Fisica, University of Padova, via Marzolo 8, 35131 Padova, Italy \\ e-mail: giovanni.picogna@pd.infn.it
}

Received 8 October 2013 / Accepted 29 January 2014

\begin{abstract}
Context. Stellar flybys in star clusters are suspected of affecting the orbital architecture of planetary systems causing eccentricity excitation and orbital misalignment between the planet orbit and the equatorial plane of the star.

Aims. We explore whether the impulsive changes in the orbital elements of planets, caused by a hyperbolic stellar flyby, can be fully damped by the circumstellar disk surrounding the star. The time required to disperse stellar clusters is comparable to the circumstellar disk's lifetime. Since we perform 3D simulations, we can also test the inclination, excitation, and damping.

Methods. We have modeled in 3D with the SPH code VINE, a system made of a solar-type star surrounded by a low density disk with a giant planet embedded in it approached on a hyperbolic encounter trajectory by a second star of similar mass and with its own disk. Different inclinations between the disks, planet orbit, and star trajectory have been considered to explore various encounter geometries. We focus on an extreme configuration where a very deep stellar flyby perturbs a Jovian planet on an external orbit. This allows us to test in full the ability of the disk to erase the effects of the stellar encounter.

Results. We find that the amount of mass lost by the disk during the stellar flyby is less than in 2D models where a single disk was considered. This is mostly related to the mass exchange between the two disks at the encounter. The damping in eccentricity is slightly faster than in 2D models and it occurs on timescales on the order of a few kyr. During the flyby both the disks are warped owing to the mutual interaction and to the stellar gravitational perturbations, but they quickly relax to a new orbital plane. The planet is quickly dragged back within the disk by the tidal interaction with the gas. The only trace of the flyby left in the planet system, after about $10^{4} \mathrm{yr}$, is a small misalignment, lower than $9^{\circ}$, between the star equatorial plane and the planet orbit.

Conclusions. In a realistic model based on 3D simulations of star-planet-disk interactions, we find that stellar flybys cannot excite significant eccentricities and inclinations of planets in stellar clusters. The circumstellar disks hosting the planets damp on a short timescale all the step changes in the two orbital parameters produced during any stellar encounter. All records of past encounters are erased.
\end{abstract}

Key words. planet-disk interactions - planet-star interactions - planets and satellites: dynamical evolution and stability hydrodynamics - methods: numerical

\section{Introduction}

Stars generally do not form alone but in bound clusters on a timescale of about 1 Myr (Hillenbrand 1997; Palla \& Stahler 2000). The embedded cluster phase lasts only a few Myr and, according to Allen et al. (2007), Fall et al. (2009), Chandar et al. (2010), and Dukes \& Krumholz (2012), after about 10 Myr, 90\% of stars born in clusters have dispersed into the field. This occurs because, after a few crossing times (Allen et al. 2007), embedded clusters lose their gaseous component, the system becomes super-virial, and the cluster rapidly dissolves. A cluster, before its dissipation, is a crowded place and its dynamical evolution is characterized by frequent stellar flybys. The close encounters can have significant effects on both disks and planetary systems surrounding the stars. They can alter the disk morphology also causing mass loss and excite planet orbits. It is expected that, in the period of higher frequency of stellar encounters, circumstellar disks are still present around stars. The lifetime of disks is comparable to the cluster bound phase being in the range 3-6 Myr, as suggested by Haisch et al. (2001), or even longer, up to 10-12 Myr, as recently argued by Bell et al. (2013). Therefore, to explore the effects of close stellar encounters on planetary systems in clusters, one has to account for the presence of circumstellar disks around the stars.
Recent $N$-body numerical modeling of the dynamical evolution of planetary systems around stars in clusters (Malmberg et al. 2011, 2007; Malmberg \& Davies 2009; Zakamska \& Tremaine 2004) have shown that scattering interactions with other stars may excite the eccentricity and inclination of planets populating the outer parts of the system. These steep changes in the orbital elements would be also observable at present and might contribute to explaining the high eccentricities of extrasolar planets discovered so far and the presence of misaligned systems. However, Marzari \& Picogna (2013) have shown that if the planet is still embedded in its birth disk during the close stellar encounter, the disk-planet tidal interaction is strong enough to effectively damp the eccentricity of the body excited by the stellar flyby on a short timescale $(\sim 10 \mathrm{kyr})$. This damping would also occur in evolved disks whose density has been significantly reduced by viscous evolution and photoevaporation. As a consequence, the coincidence of the cluster lifetime with that of the circumstellar disks plays against a significant role of stellar flybys in affecting the present dynamical architecture of the planet.

The simulations presented in Marzari \& Picogna (2013) were based on the 2D code FARGO and considered a single disk around the star with planets while the perturber star was assumed to be diskless. In this paper we extend that model using a 
3D SPH code and including two disks, one around each star. This allows us to model more accurately close stellar flybys where the two disks closely interact. In addition, we can also explore the excitation of the planet inclination and its subsequent damping due to the interaction with the disk (Marzari \& Nelson 2009; Bitsch \& Kley 2011; Cresswell et al. 2007). We concentrate in this paper on the effects of a single deep close stellar encounter on a planet still embedded in its birth disk. If the effects of an extremely close flyby can be erased by the disk, we expect that the cumulative effects of multiple but more distant encounters can also be absorbed by the disk. We will also explore the effects on the disk morphology of the stellar tidal force and of the mutual disk interaction during the encounter.

The paper is organized as follows. In Sect. 2 we describe the numerical algorithm used to model the disk and planet evolution during and after a hyperbolic close encounter with another star. In Sect. 3 we outline the different flyby configurations we have modeled while Sects. 4-6 are devoted to the presentation of the numerical outcomes. In Sect. 7 we discuss our results and their implications for the evolution of planetary systems in stellar clusters.

\section{The numerical model}

The hydrodynamical code used to model the evolution of the disks surrounding both stars is VINE (Wetzstein et al. 2009; Nelson et al. 2009). It is based on the smoothed particle hydrodynamics (SPH) algorithm, which solves the hydrodynamical equations by replacing the fluid with a set of particles (Gingold \& Monaghan 1977). The code has been updated to improve momentum and energy conservation as described in Picogna \& Marzari (2013). Variable smoothing and softening lengths have been introduced as in Price \& Monaghan (2004), Springel \& Hernquist (2002), and Price \& Monaghan (2007). To realistically model the gas accretion onto the planet, we implemented the algorithm described in Ayliffe \& Bate (2009). The planet potential is modified adding a surface potential term,

$$
F_{r}=-\frac{G M_{\mathrm{p}}}{r^{2}}\left[1-\left(\frac{2 R_{\mathrm{p}}-r}{R_{\mathrm{p}}}\right)^{4}\right],
$$

for $r<2 R_{\mathrm{p}} ; M_{\mathrm{p}}$ and $R_{\mathrm{p}}$ are the initial mass and radius of the planet, respectively. When an SPH particle reaches the surface of the planet $\left(R_{\mathrm{p}}\right)$ the net force is 0 , whereas if $r$ becomes smaller than $R_{\mathrm{p}}$, the potential becomes strongly repulsive and the particle is driven back to the planet surface.

The disk around each star has an initial density at 1 AU of $\rho_{0} \sim 1 \times 10^{-11} \mathrm{~g} / \mathrm{cm}^{3}$, approximately $1 / 100$ of the minimum mass solar nebula (MMSN). Its radius extends out to $40 \mathrm{AU}$ with a density profile declining as $\rho(r)=\rho_{0} r^{-3 / 2}$, corresponding to a superficial density decreasing as $\Sigma=\Sigma_{0} r^{-1 / 2}$. The total mass of each disk is $7 \times 10^{-3} M_{\odot}$, about 14 times less massive than the disks modeled in Forgan \& Rice (2009). The reason for such a low density is justified by the presence of a fully formed giant planet in the disk. In our scenario, core-accretion and gas infall have already occurred and most of the gas is expected to have been either accreted by the star because of viscous evolution or dispersed by photo-evaporation. In addition, if eccentricity and inclination damping occurs in light disks, it would be even more efficient in massive disks. In modeling these light disks we can neglect the effects of self-gravity and adopt the isothermal approximation since they are optically thin. The disk scale height $H$ is set to 0.05 giving a temperature $T_{0}$ at $1 \mathrm{AU}$ from the star of about $620 \mathrm{~K}$. During the stellar encounter the disks may become warped, and so the isothermal temperature profile is always computed with respect to the average median plane of the disk. Each disk is simulated with $850000 \mathrm{SPH}$ particles for a total of 1.7 million particles for the whole system.

We adopted the standard SPH artificial viscosity (Monaghan $\&$ Gingold 1983), introducing a linear $\left(\alpha_{\mathrm{SPH}}\right)$ and a quadratic $\left(\beta_{\mathrm{SPH}}\right)$ term whose initial values are set to 0.1 and 0.2 , respectively. Meru \& Bate (2012) showed that these terms can be compared to the Shakura \& Sunyaev (1973) $\alpha$-viscosity parameter $\alpha_{\mathrm{SS}}$ defined as $v=\alpha_{\mathrm{SS}} c_{\mathrm{S}} H$ where $v$ is the kinematic viscosity, $c_{\mathrm{S}}$ is the sound speed, and $\mathrm{H}$ is the vertical pressure scale height. Using their relations we find that, away from the close approach, the corresponding $\alpha_{\mathrm{SS}}$ in our models is about 0.002 .

One disk harbors a Jupiter-sized planet orbiting at $18 \mathrm{AU}$ on a circular orbit not inclined with respect to the disk. We set the planet on an outside trajectory since we want to maximize the effects of the stellar encounter in order to test if, in spite of the highly perturbing configuration, the disk is still able to damp the planet's eccentricity and inclination after the flyby. The second star is started on a hyperbolic orbit with a minimum impact parameter $q$ fixed at the beginning of the simulation. The initial distance between the two stars is $450 \mathrm{AU}$ (approximately $500 \mathrm{yr}$ before the encounter) and the relative velocity at infinity is set to $1 \mathrm{~km} \mathrm{~s}^{-1}$, a typical value in clusters. Different inclinations are considered for the passing star to test the amount of disk warping and planet excitation in inclination. The two disks are oriented at different angles up to the extreme case of prograde-retrograde encountering disks where one is rotating in the direction of the encounter orbit, and the other against it.

Prior to the encounter, the planet begins to carve a gap. We might have let the disk-planet system evolve longer in order to allow the planet to create a full gap. However, as also shown in Fragner \& Nelson (2009) and Marzari \& Picogna (2013), a close stellar encounter strongly perturbs the disk and planetary orbit, cancelling out any pre-existing structure including a gap around the planet. A new gap is created some time after the stellar flyby when the disk relaxes, as shown in our simulations.

\section{Definition of the different flyby geometry and disk inclinations}

Owing to the large amount of CPU time required by each 3D run, we have performed a limited number of simulations covering, by necessity, a small but meaningful portion of the initial phase space. We look for the most perturbed configurations since our goal is to test the ability of the gaseous disk to damp the planet orbital parameters after the stellar encounter even in extreme conditions. In Table 1 we summarize the main initial parameters of each simulation (Cols. 1-5 of the table) while the changes in some relevant disk properties during the evolution of the system are given in the other columns. Their meaning will be described later on.

To compute the initial set up for the disks and the hyperbolic encounter, we start from a coplanar configuration where the hyperbolic trajectory of the second star has the pericenter on the positive $x$-axis while the star harbouring the planet is at the origin of the $x-y$ frame. We then tilt the hyperbolic orbit around the $x$-axis by an angle $i_{\mathrm{s} 2}$ to vary the approach geometry. We also rotate the disk of the second star around the $y$-axis by an angle $i_{\mathrm{d} 2}$. The disk of the primary star has instead its median plane on the $x-y$ plane. We could also have varied the initial configuration by rotating both the disks around the $x$ - and $z$-axes, but because of the heavy CPU load of each simulation, we considered only the rotations defined by $i_{\mathrm{s} 2}$ and $i_{\mathrm{d} 2}$. 
Table 1. List of models.

\begin{tabular}{ccccccccccccc}
\hline \hline $\mathrm{id}$ & $i_{\mathrm{d} 2}$ & $i_{\mathrm{s} 2}$ & $q(\mathrm{AU})$ & $\mathrm{nd}$ & $\frac{\Delta M_{\mathrm{d} 1}}{M_{\mathrm{d} 1}}$ & $\frac{\Delta M_{\mathrm{d} 2}}{M_{\mathrm{d} 2}}$ & $M_{12}$ & $M_{21}$ & $e_{\mathrm{d} 1}$ & $e_{\mathrm{d} 2}$ & $i_{\mathrm{d} 1}$ & $i_{\mathrm{d} 2}$ \\
\hline $\mathrm{a}$ & $45^{\circ}$ & $0^{\circ}$ & 55 & 2 & $-16 \%$ & $-3 \%$ & $11 \%$ & $10 \%$ & 0.09 & 0.09 & $2.6^{\circ}$ & $-3.4^{\circ}$ \\
$\mathrm{b}$ & $45^{\circ}$ & $0^{\circ}$ & 65 & 2 & $-11 \%$ & $-1 \%$ & $7 \%$ & $6 \%$ & 0.05 & 0.05 & $1.0^{\circ}$ & $-1.8^{\circ}$ \\
$\mathrm{c}$ & $45^{\circ}$ & $60^{\circ}$ & 55 & 2 & $-2 \%$ & $-17 \%$ & $3 \%$ & $13 \%$ & 0.04 & 0.07 & $8.5^{\circ}$ & $2.0^{\circ}$ \\
$\mathrm{d}$ & $0^{\circ}$ & $0^{\circ}$ & 55 & 2 & $-6 \%$ & $-5 \%$ & $8 \%$ & $11 \%$ & 0.08 & 0.09 & $0.0^{\circ}$ & $0.0^{\circ}$ \\
$\mathrm{e}$ & $\ldots$ & $0^{\circ}$ & 55 & 1 & $-21 \%$ & $\ldots$ & $15 \%$ & $0 \%$ & 0.08 & 0.31 & $0.0^{\circ}$ & $0.0^{\circ}$ \\
$\mathrm{f}$ & $180^{\circ}$ & $0^{\circ}$ & 55 & 2 & $-24 \%$ & $2 \%$ & $8 \%$ & $2 \%$ & 0.07 & 0.17 & $0.0^{\circ}$ & $0.0^{\circ}$ \\
$\mathrm{g}$ & $60^{\circ}$ & $30^{\circ}$ & 55 & 2 & $-10 \%$ & $-8 \%$ & $9 \%$ & $11 \%$ & 0.06 & 0.05 & $9.2^{\circ}$ & $58.9^{\circ}$ \\
$\mathrm{h}$ & $-60^{\circ}$ & $30^{\circ}$ & 55 & 2 & $-17 \%$ & $2 \%$ & $10 \%$ & $5 \%$ & 0.06 & 0.06 & $6.0^{\circ}$ & $61.6^{\circ}$ \\
\hline
\end{tabular}

Notes. Each simulation is labeled with a letter in Col. $1 ; i_{\mathrm{d} 2}$ is the mutual inclination between the two disks defined by a rotation along the $x$-axis; $i_{\mathrm{s} 2}$ is the inclination of the hyperbolic trajectory of the second star computed with respect to the initial plane of the primary disk; $q$ is the minimum approach distance during the flyby; and $n d$ indicates the number of disks included in the simulation. At the end of each simulation we computed the amount of mass lost by disk $1 \frac{\Delta M_{\mathrm{d} 1}}{M_{\mathrm{d} 1}}$ and disk $2 \frac{\Delta M_{\mathrm{d} 2}}{M_{\mathrm{d} 2}} . M_{12}$ and $M_{21}$ are the amounts of mass transferred from one disk to the other. $e_{\mathrm{d} 1}, e_{\mathrm{d} 1}, i_{\mathrm{d} 1}$, and $i_{\mathrm{d} 1}$ are the disk eccentricities and inclinations.
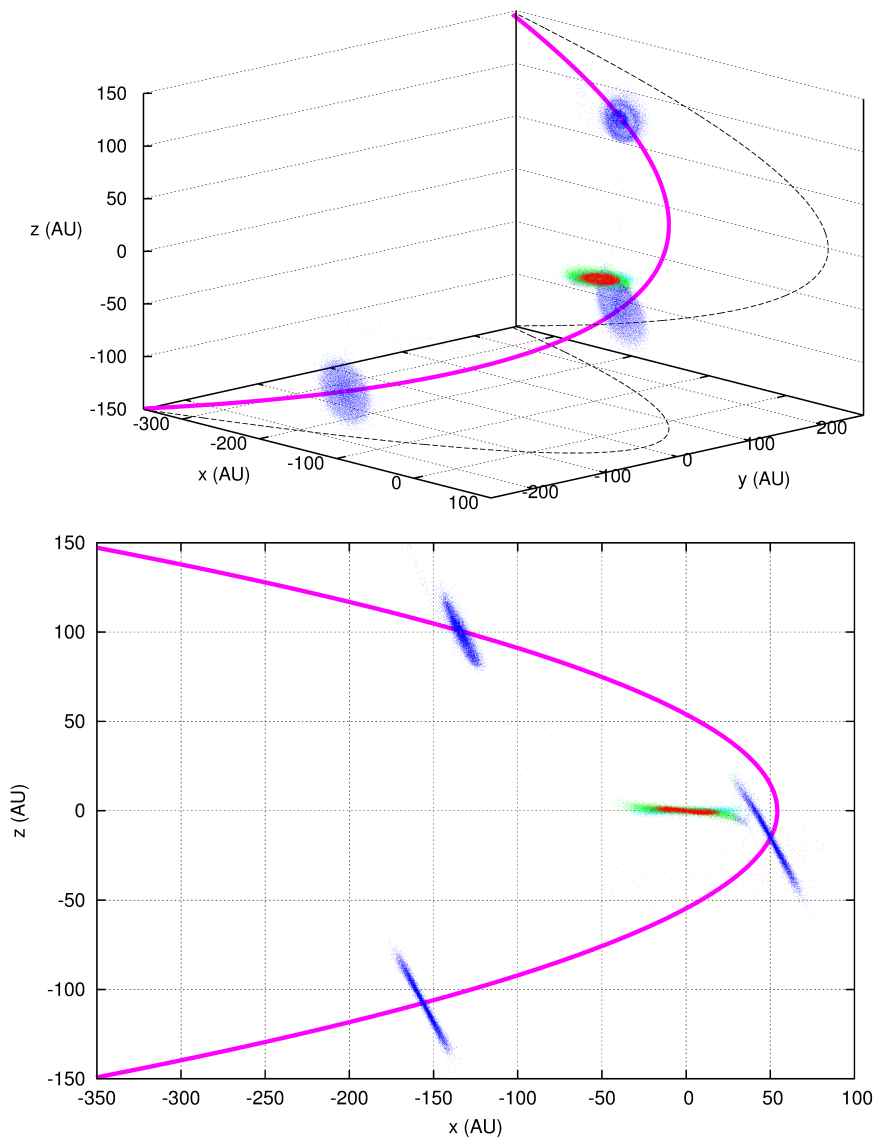

Fig. 1. Top panel: 3D illustration of the encounter geometry of model h. The hyperbolic trajectory is plotted as a continuous line while its projections on the $(x, y)$ and $(x, z)$ plane are shown as dashed lines. The disks around the primary and secondary star are shown at three different evolutionary times around the pericenter passage. The primary disk is at the center of the reference frame so its shape overlaps at the three different times. Bottom panel: projection of the 3D plot on the $(x, z)$ plane, a view that helps to understand the dynamical configuration of the encounter.

In Fig. 1a we show an example of this procedure giving a full $3 \mathrm{D}$ view of the encounter geometry for the case $\mathrm{h}$ where the disk has an inclination of $60^{\circ}$ with respect to the primary disk and the hyperbolic trajectory is inclined of $30^{\circ}$ with respect to the primary disk plane. The hyperbolic trajectory is shown during the pericenter passage and the two disks are plotted at three different evolutionary times. A 2D projection in the $(x, z)$ plane (Fig. 1b) of the previous plot gives an additional view of the mutual orientations of stars and disks.

In a test case we changed the minimum approach distance $q$ (case b) and we also performed a simulation with no disk around the second star to compare with all other cases with two disks (case d). This model is relevant for understanding the role of the disk around the second star in the evolution of the planet.

\section{Cases a, b, and c: $i_{\mathrm{d} 2}=45^{\circ}$}

We first analyze the models where the disks are mutually inclined by $45^{\circ}$, i.e. $i_{\mathrm{d} 2}=45^{\circ}$. In two cases with this configuration we test the dependence on the pericenter distance $q$ of the hyperbolic orbit on the evolution of the disks and planet orbit (cases a and $\mathrm{b}$ ). In the third case $\mathrm{c}$ we also incline the hyperbolic trajectory setting $i_{\mathrm{s} 2}=60^{\circ}$. This last case is more general in terms of geometric configuration and we will adopt it as a reference case comparing its outcome to the other two. In Fig. 2 the evolution of the disks and planet are shown at different times for case c. At the minimum distance (first two plots) the disks begin to interact becoming highly eccentric and strongly warped. The outer edge of the disk around the passing star overlaps close to the center with that of the primary star. At subsequent times, the disks are very distorted, and tidal tails are launched causing a significant mass exchange. It is noteworthy that in the third plot the disk around the passing star (secondary star) appears very eccentric, but this is an effect of perspective. When the integrated density is computed, the inclined disk is projected on the $x-y$ plane and so it appears more eccentric because of its inclination. About $2800 \mathrm{yr}$ after the flyby, the primary disk is returned to a quiet state and the planet has carved a new gap in the disk, as illustrated in Fig. 3.

\subsection{Evolution of the planet orbit}

The planet in case c, which appears in the plots of Figs. 2 and 3 as a local overdensity, moved to an inclined and eccentric orbit after the stellar flyby owing to the gravitational pull of the passing star. However, the subsequent interaction with the disk damps both the eccentricity and inclination and, on a short timescale, the planet is driven back on an almost circular orbit not inclined with respect to the disk plane. All the effects of the stellar flyby have been erased apart from a shift in semi-major axis. However, the final planet orbit may be slightly inclined with respect to 

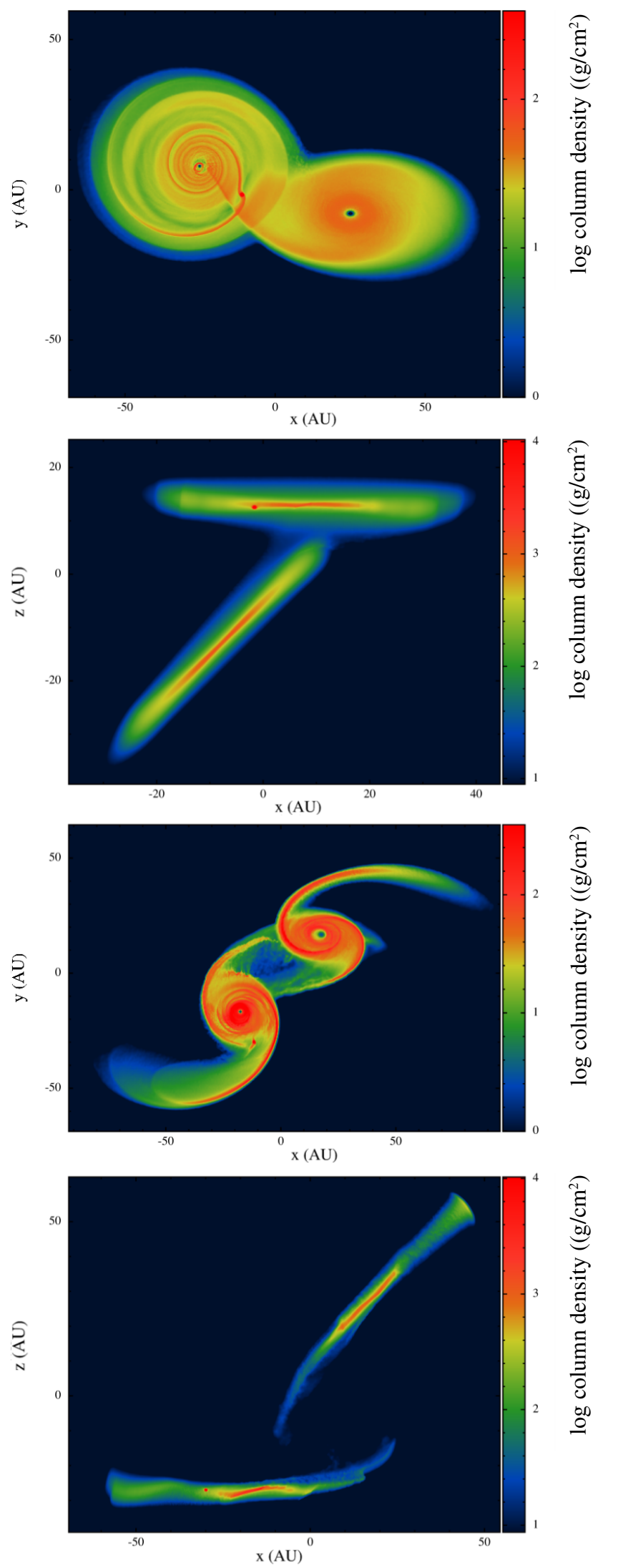

Fig. 2. Logarithm of the superficial density (column integrated) of the circumstellar disks in the $x-y$ and $x$ - $z$ plane, at two different evolutionary steps during the stellar flyby for the model c. We note that the color scale for the superficial density $\Sigma$ is different in the $x-y$ (face-on) and $x-z$ (edge-on) projections since the bulk density $\rho$ is integrated along different paths. The top plots show the disks when the stars are close to the pericenter passage, the bottom plots when the stars are departing just $60 \mathrm{yr}$ after the encounter.
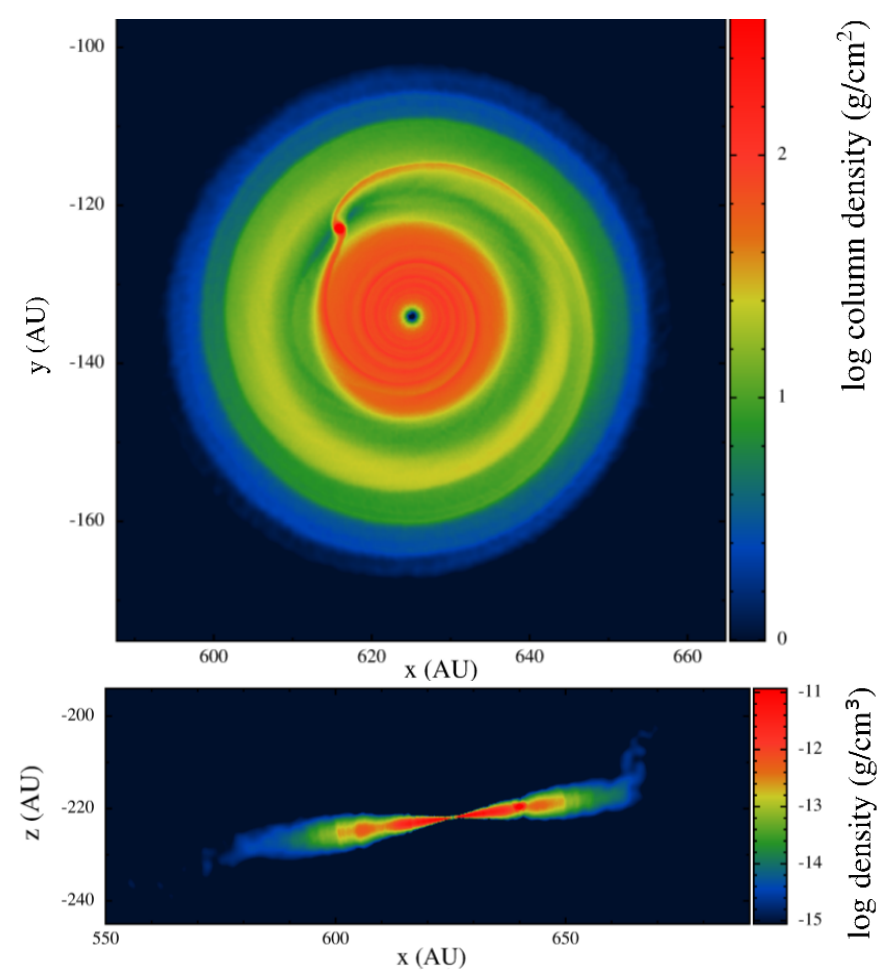

Fig. 3. Logarithm of the superficial density (column integrated) of the disk around the star with a planet and its $x-z$ slice $2800 \mathrm{yr}$ after the flyby for the model c. The disk has reached a quiet state and the planet has almost completely carved a gap around its orbit.

the equatorial plane of the primary star since the disk, after a warped phase, relaxes into an inclined configuration (see Fig. 3, bottom plot). The planet interacts tidally with the disk and is not dragged back to the star's equatorial planet but to the disk's median plane, slightly tilted with respect to the star's equatorial planet as a result of the flyby.

In Fig. 4 we show the time evolution of the orbital parameters of the planet in the three simulations $\mathrm{a}, \mathrm{b}$, and $\mathrm{c}$. The semi-major axis, after a jump caused by the stellar encounter, decreases with time because of the tidal interaction with the disk. A new gap around its orbit is forming, as shown in Fig. 3 (top plot) for the case $\mathrm{c}\left(i_{\mathrm{d} 2}=45^{\circ}\right.$ and $\left.i_{\mathrm{s} 2}=60^{\circ}\right)$, but it is not yet deep enough to cause the slow type I migration which will probably start at a later time. The delay is also due to the initially inclined orbit of the planet with respect to the disk which slows down the gap carving.

The eccentricity pumped up by the stellar flyby is quickly damped when the second star departs from the primary after the encounter. This is illustrated in Fig. 4, middle panel. In the most perturbed configuration (case a) the eccentricity grows up to 0.5 and is quickly reduced to 0.2 in less than $1000 \mathrm{yr}$. After this initial phase, the damping is slower, occurring at a rate of about $6 \times 10^{-6} \mathrm{yr}^{-1}$ and leading to a full damping on a timescale, in the worst case, of a few $10^{4} \mathrm{yr}$. In cases $\mathrm{b}$ and $\mathrm{c}$ the initial eccentricity excitation is of the same order of magnitude. The inclination of the hyperbolic orbit of case c possibly reduces the strength of the passing star perturbations on the planet eccentricity as does the larger flyby minimum distance in case b. However, the damping appears to be faster in case c where the final eccentricity, after $2700 \mathrm{yr}$ from the flyby, is only 0.02 .

In case $c$ the inclination of the planet with respect to the equatorial plane of its host star is excited during the flyby up to about $4.5^{\circ}$. After the stellar encounter the disk is also inclined 
G. Picogna and F. Marzari: Circumstellar disks and stellar flybys

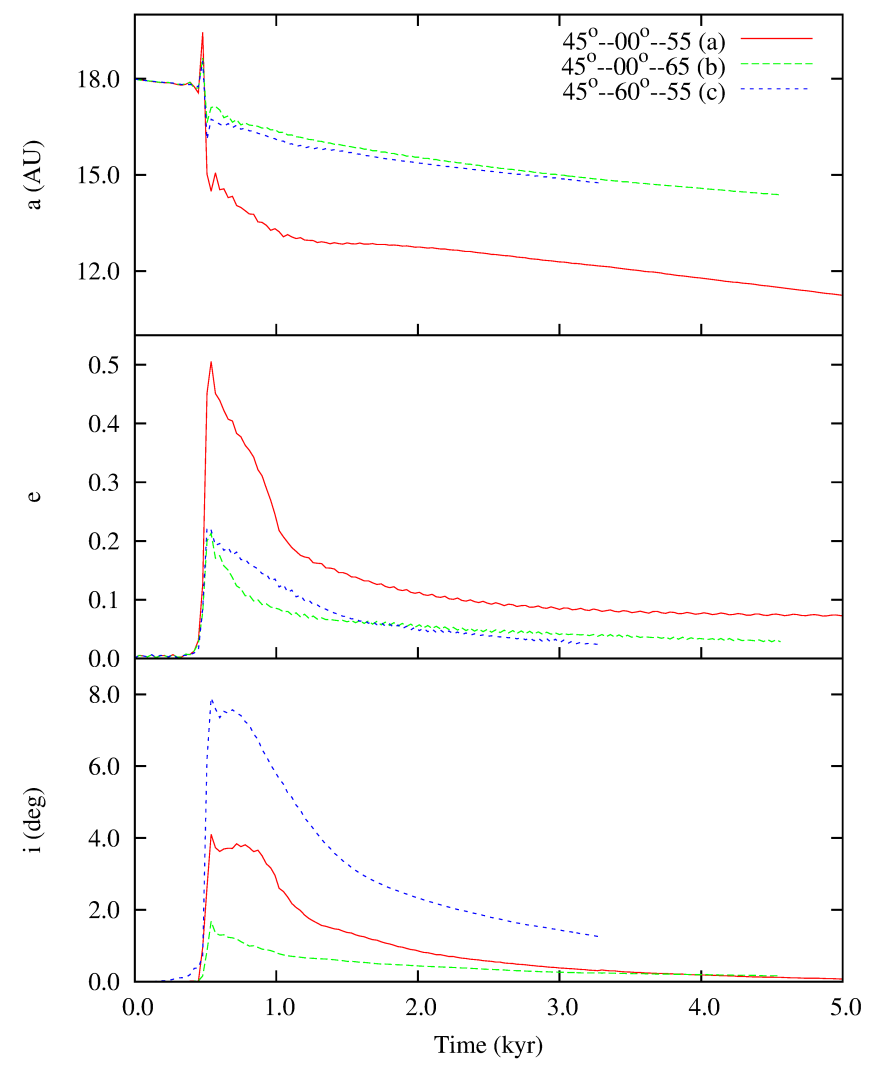

Fig. 4. Evolution of the semi-major axis (top panel), eccentricity (middle panel), and inclination (bottom panel) of the planet in models $\mathrm{a}, \mathrm{b}$, and $\mathrm{c}$.

with respect to its initial plane and its inclination is about $8.5^{\circ}$ as shown in Fig. 5, top plot. The gravitational interaction between the planet and disk drives the planet back into the disk and its inclination is slowly brought to the same value as that of the disk. The mutual inclination between the planet and the disk decreases smoothly with time to a low value of about $1^{\circ}$ when the simulation was stopped after $2.3 \mathrm{kyr}$. In Fig. 5, bottom plot, we show the evolution of the disk and planet inclinations in case a. The orbit of the planet is not excited in inclination by the stellar flyby since $i_{\mathrm{s} 2}=0$. However, the disk around the primary star is first warped and then it settles down to an inclination of about $2.8^{\circ}$. The planet inclination grows until the mutual inclination between planet and disk is negligible.

The outcome of these models confirms that the presence of the disk leads to a fast damping of the eccentricity and inclination due to the stellar flyby, erasing any trace of the event in the trajectory of the planet. Such an event might be recorded in the inclination of the disk (and then of the planet) with respect to the equatorial plane of the star, but the expected tilting would be very small. It could accelerate the inward migration of the planet by restarting type I migration after the stellar flyby when the gap is erased, but soon a new gap would be cleared and type II migration restored.

\section{Coplanar cases $d, e$, and $f$}

These models assume a coplanar initial configuration for the disks and for the hyperbolic orbit of the passing star, i.e. $i_{\mathrm{d} 2}=0^{\circ}$ and $i_{\mathrm{s} 2}=0^{\circ}$. They are analogous to $2 \mathrm{D}$ models except that the disks are computed in 3D. We do not have changes in inclination and we concentrate only on the semi-major axis and eccentricity

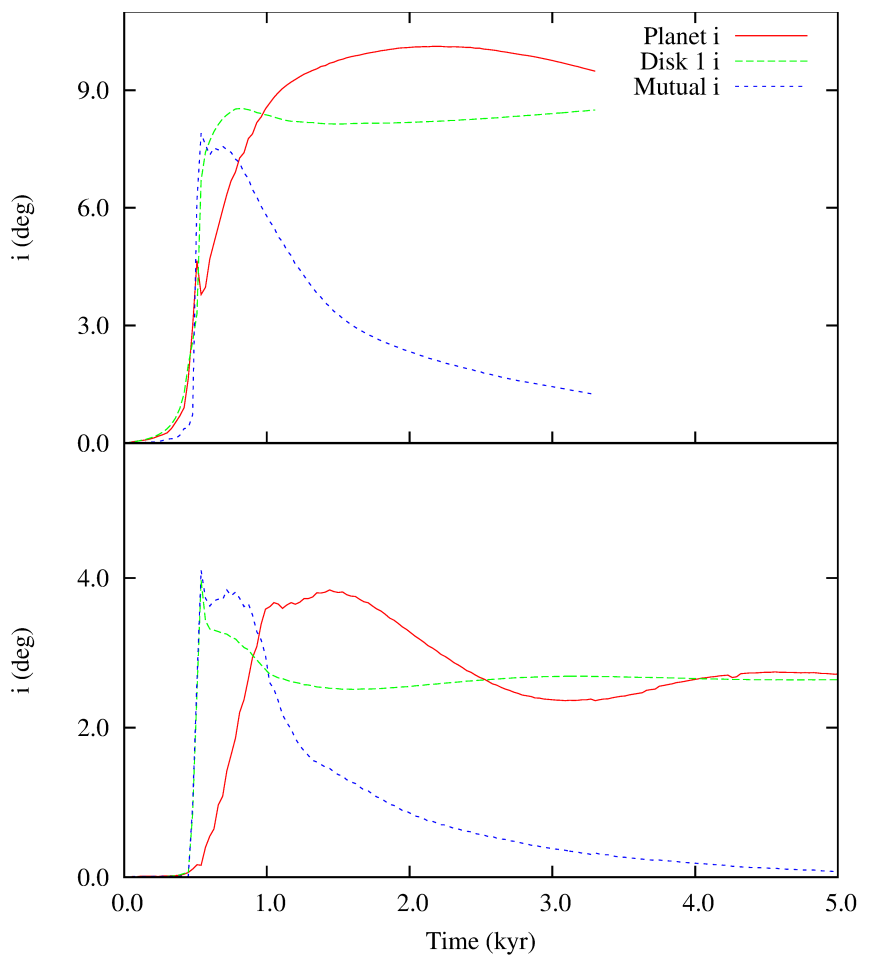

Fig. 5. Evolution of the disk, planet, and mutual inclination for cases $\mathrm{c}$ (top panel), and a (bottom panel).

variations. As illustrated in Fig. 6, the eccentricity and semimajor axis jumps in the three cases $\mathrm{d}$, e, and $\mathrm{f}$ are about the same. This confirms that the gravitational perturbations of the passing star dominates the dynamical evolution of the planet during the flyby. The subsequent damping is not significantly influenced by the different evolution and mass loss of the disk (see Table 1), but it is slightly faster compared to that of case a $\left(i_{\mathrm{d} 2}=45^{\circ}\right.$, $i_{\mathrm{s} 2}=0^{\circ}$ ), shown as a reference in Fig. 6 . This is possibly due to the disk-planet mutual inclination that, in case a, reduces the tidal interaction.

We considered the prograde-retrograde encountering configuration as an extreme case with one disk (that hosting the planet) rotating in the direction of the encounter orbit, and the other rotating against the encounter. The star is on a hyperbolic orbit coplanar to both disks. The evolution of the disks is not symmetric since for one disk (the primary) the secondary star is on a prograde trajectory while for the second disk the primary star is seen to be moving on a retrograde trajectory. In Fig. 10 the disk integrated column density is shown shortly after the stellar flyby (top panel) and 60 years later (bottom panel). The two disks are not perturbed in a symmetric way, as for the coplanar prograde case, and only the primary shows a consistent mass loss along the trailing tidal wave. The disk around the secondary passing star does not become significantly elliptic and it does not develop tidal waves before the encounter. It appears to be less perturbed by the primary star gravity and this is in agreement with the findings of Hall et al. (1996) claiming that the angular momentum transfer between a perturber and a disk in a retrograde configuration is smaller. A similar effect was also observed in Forgan \& Rice (2009) for more massive disks. At the pericenter of the flyby the two disks come in contact and mass is transferred from one to the other. As can be noted in Fig. 10, the primary disk shows the usual spiral structure with the trailing arm extending into the secondary disk. Because of the direct 


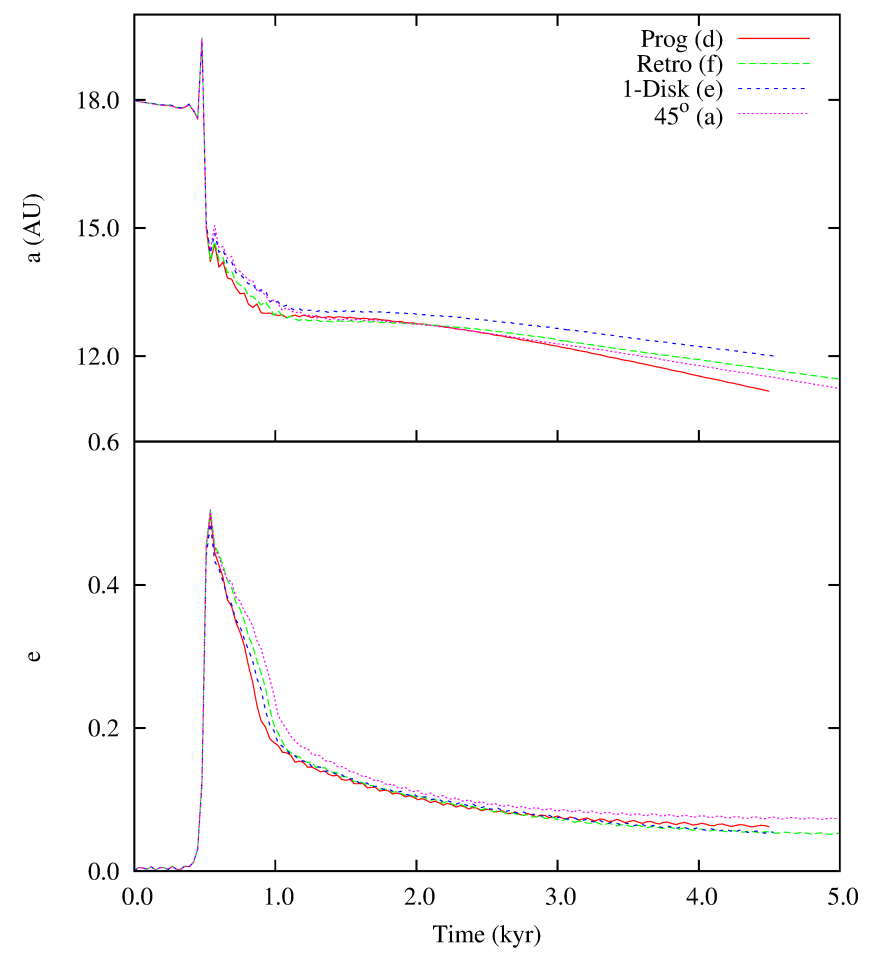

Fig. 6. Evolution of the planet's orbital elements $a$ and $e$ in models d, e, and $\mathrm{f}$ (coplanar cases), compared with case a.

interaction between the disks, the upper part of the secondary disk (retrograde with respect to the primary star) is strongly distorted while the bottom part of the disk is almost circular and unperturbed. According to Table 1 the primary disk loses approximately $25 \%$ of its mass while the secondary disk gains $2 \%$ of its initial mass.

In spite of the strong differences in the disk evolution between the prograde and retrograde cases, the orbital evolution of the planet is not significantly different. This reinforces the idea that the tidal damping of the planet eccentricity is a robust mechanism that does not depend significantly on the disk evolution. Once the perturbing effects of the star flyby are passed, and this occurs on a short timescale, the disk relaxes and drives the planet back to a circular non-inclined orbit.

\subsection{Mass exchanged by disks or lost to infinity}

A significant difference between the 2D models of Marzari \& Picogna (2013) and the 3D results presented here is the amount of mass lost by the disk with a planet during the encounter. In Marzari \& Picogna (2013) the primary disk retained about half of its initial mass at the end of the simulation while in the 3D cases presented here the fraction of mass loss, summarized for all models in Table 1, is significantly smaller and it strongly depends on the encounter geometry, as shown in Fig. 8. This difference may be ascribed to the following reasons.

- In previous 2D models only one disk was included while in the present models we consider two disks. The presence of the disk around the secondary star has important consequences for the evolution of the system since a consistent fraction of the mass lost during the encounter by the disk around the primary is replaced by mass stripped from the disk of the secondary. According to Table 1 up to $13 \%$ of the disk's initial mass can be exchanged between the two

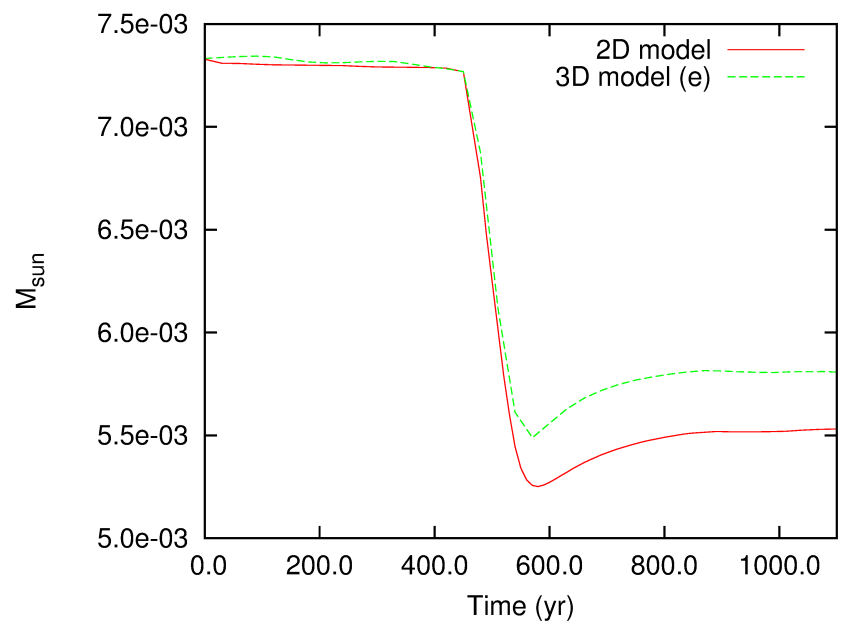

Fig. 7. Disk mass evolution in the 3D model e compared to a 2D model with identical initial parameters and integrated with the same SPH code VINE. The mass loss during the encounter is higher in the 2D model.

disks. Explicative in this context is the outcome of model e. In Fig. 9 we show the density distribution after the stellar encounter illustrating how, during the stellar flyby, the second star, initially without its own disk, strips material from the primary. The new captured disk has a mass equal to $15 \%$ of the initial disk mass of the primary (see Table 1). This proves that a large amount of mass can be transferred from one disk to the other, an effect not observed in Marzari \& Picogna (2013) because the secondary star was out of the computational grid.

- In 3D the disk evolution, and then the mass loss, also depends on the behaviour along the vertical direction. This leads to a lower mass loss in $3 \mathrm{D}$ models with respect to 2D models as illustrated in Fig. 7 where we compare the outcome of the 3D model e with a 2D simulation, performed with the SPH code, having the same initial physical parameters and profiles as model e. The mass loss is slightly higher in the 2D model just after the stellar flyby. However, the comparison also suggests that changing the dimensions of the simulations has less impact on the mass loss than the existence of a secondary disk. As a consequence, the mass loss difference between 2D and 3D models is mainly due to the mass exchange between the two circumstellar disks. We note that the apparent increase of the disk mass after it hit the minimum is fictitious and is due to the difficulty of defining the disk borders during the encounter when the shape of the disk is very elongated and asymmetrical.

- Previously published 2D simulations were performed with a grid code having a limited spatial domain centered on the primary star. Mass exiting the grid domain was lost by the system. In the models shown here an SPH code is used and there are no boundaries for the disks. As a consequence, gas excited on highly eccentric trajectories during the stellar flyby is allowed to fall back on the disk at later times while in the simulations with the grid code it was lost. This can also be seen in Fig. 8 where the mass of the disk is computed by adding up all the mass moving within $R_{\text {out }}=50 \mathrm{AU}$ from the star. During the flyby mass is lost, but part of it comes back within $R_{\text {out }}$ before the onset of an almost constant mass loss due to viscosity, spiral waves excited by the planet, and residual disk instability related to the stellar flyby. 


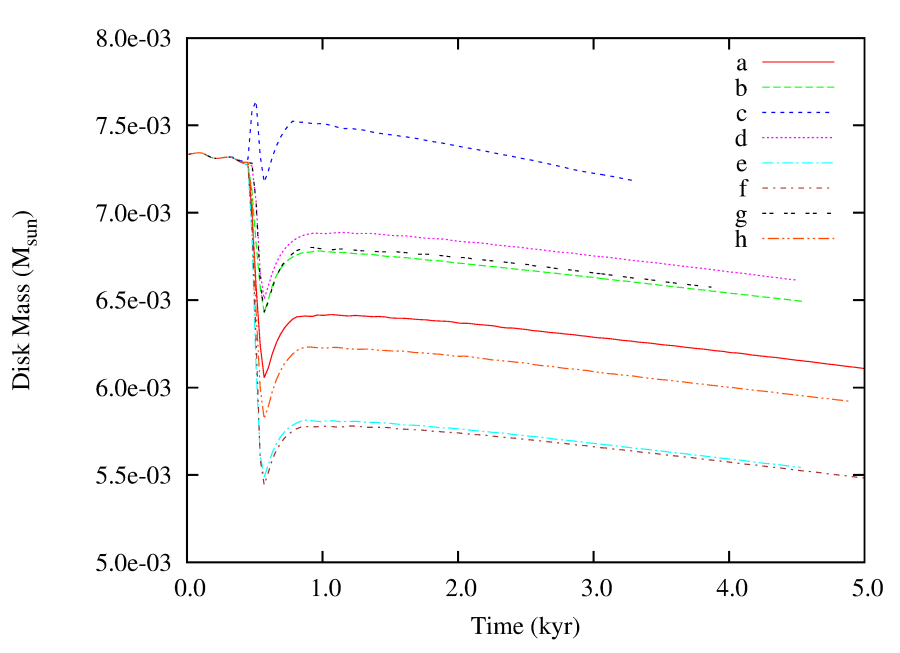

Fig. 8. Mass of the disk around the star harbouring the planet as a function of time for the different models summarized in Table 1. The flyby is marked by a jump followed by a slow mass decrease.

- The 2D models were propagated a bit longer in time and this might explain part of the larger mass loss. In the 3D models the disk is still losing a small amount of mass when we halt the simulation. This last effect, however, accounts for only a small fraction of the difference in the mass loss between 2D and 3D models.

Since the aim of our calculations is to show that the disk damps the excitation caused by the stellar flyby on the planet orbital elements, we are only interested in the mass loss that occurs during the damping. The disk, on this short timescale ( $\sim 5 \mathrm{kyr})$, possibly does not reach a configuration where the mass loss is only due to viscosity and spiral waves excited by the planet, but it is still losing mass because of the binary perturbations. However, this is not relevant for our computations since the damping of the planet orbit has already occurred.

The mass exchange might cause a limited variation in the original metallicity of the disk compared to that of the host star assuming that the two encountering stars have significantly different values of metallicity. However, the amount of pollution is tiny and possibly without significant consequences for planet formation. The reduced mass loss in the more realistic 3D models presented here leads to a longer lifetime of the disk and a stronger damping effect on the eccentricity and inclination of the planet after the flyby.

\section{Highly misaligned configurations: cases $\mathrm{g}$ and $\mathrm{h}$}

We finally considered two cases with a high mutual inclination between the disks and an inclined hyperbolic trajectory for the star. In cases $\mathrm{g}$ and $\mathrm{h}, i_{\mathrm{d} 2}=60^{\circ}$ and $i_{\mathrm{d} 2}=-60^{\circ}$, respectively, while $i_{\mathrm{s} 2}=30^{\circ}$. In Fig. 11 we illustrate the orbital evolution of the planet. The evolution of the semi-major axis and eccentricity is very similar to that of the other cases previously discussed. It is noteworthy that the initial eccentricity step is inversely proportional to $i_{\mathrm{s} 2}$, the inclination of the hyperbolic orbit with respect to the primary disk plane. When $i_{\mathrm{s} 2}=0^{\circ}$ the jump is about 0.5 , for $i_{\mathrm{s} 2}=30^{\circ}$ it is slightly smaller than 0.4 , while it reduces to 0.2 when $i_{\mathrm{s} 2}=60^{\circ}$. A similar trend was also observed by Forgan \& Rice (2009) and it may then be unnecessary to test more highly inclined configurations since the $\Delta e$ would be smaller.

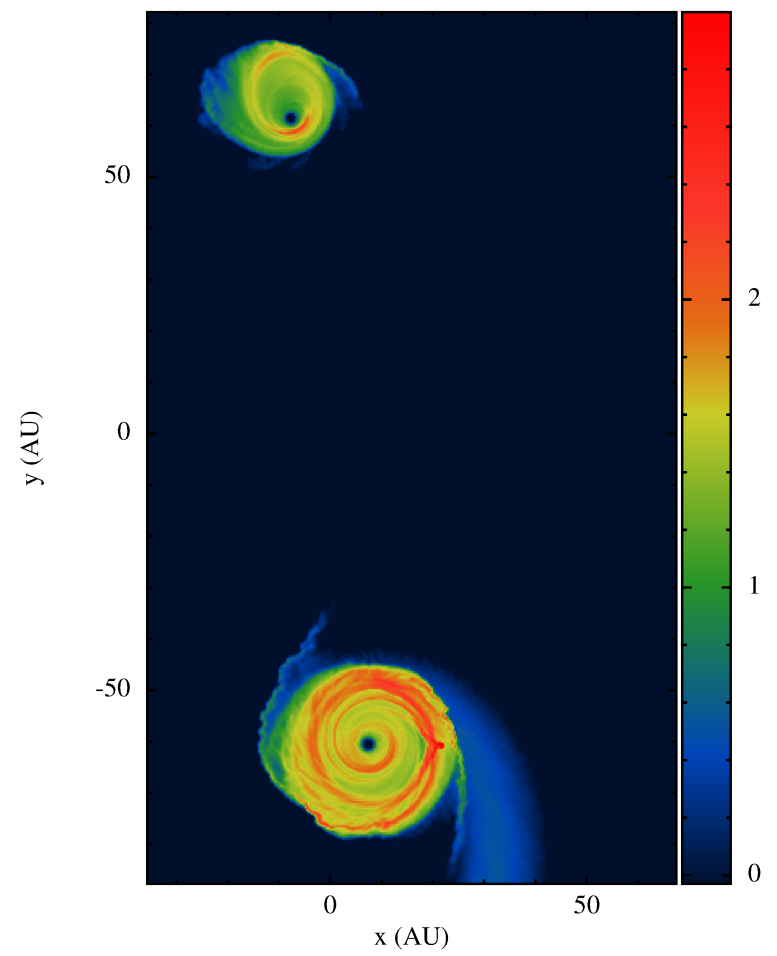

Fig. 9. Capture of a disk by the second star, initially without any disk (model e), during the stellar flyby. The plot shows the system shortly after the stellar encounter when the material has been transferred from one star to the other via the extended tidal wave.

A small difference between cases $g$ and $h$ in terms of eccentricity damping rate can be ascribed to the different mass loss of the two disks. In case $\mathrm{g}$ the disk mass is reduced by $10 \%$ after about 3000 yr while in case $\mathrm{h} 17 \%$ of the mass is lost after the same time interval (Table 1).

As in case $\mathrm{c}$, the mutual inclination between the planet and disk is reduced and the planet is quickly pulled back into the disk (Fig. 11, bottom plot). Even in this highly inclined configuration, the maximum inclination of the disk (and planet) around the primary star is excited to a maximum of about $9^{\circ}$ (case g) which will be the final misalignment between the equatorial plane of the star and the final planet orbit after the disk dissipation.

\section{Conclusions}

The 3D simulations of stellar encounters in the presence of circumstellar disks presented in this paper confirm that the orbital changes a planet undergoes during a close stellar flyby are quickly damped by the interaction with the disk. The only observable difference after the close approach may be a residual misalignment between the planet-disk orbital plane and the star equatorial plane. During the encounter, the disk harbouring the planet is warped by the interaction with the passing star and its disk. Gradually, it relaxes on a new plane slightly tilted with respect to the original one. The amount of tilting depends on the geometrical configuration of the system prior the encounter and in our models we find a maximum value of about $9^{\circ}$. Subsequently, the planet is pulled back into the disk by the tidal interaction with the gas and its orbit also becomes inclined with respect to the star equatorial plane. In case of repeated stellar flybys the final tilting of the planet orbit may be higher than $9^{\circ}$, but it appears unlikely that this mechanism is responsible for the high planet inclinations found in some extrasolar systems 

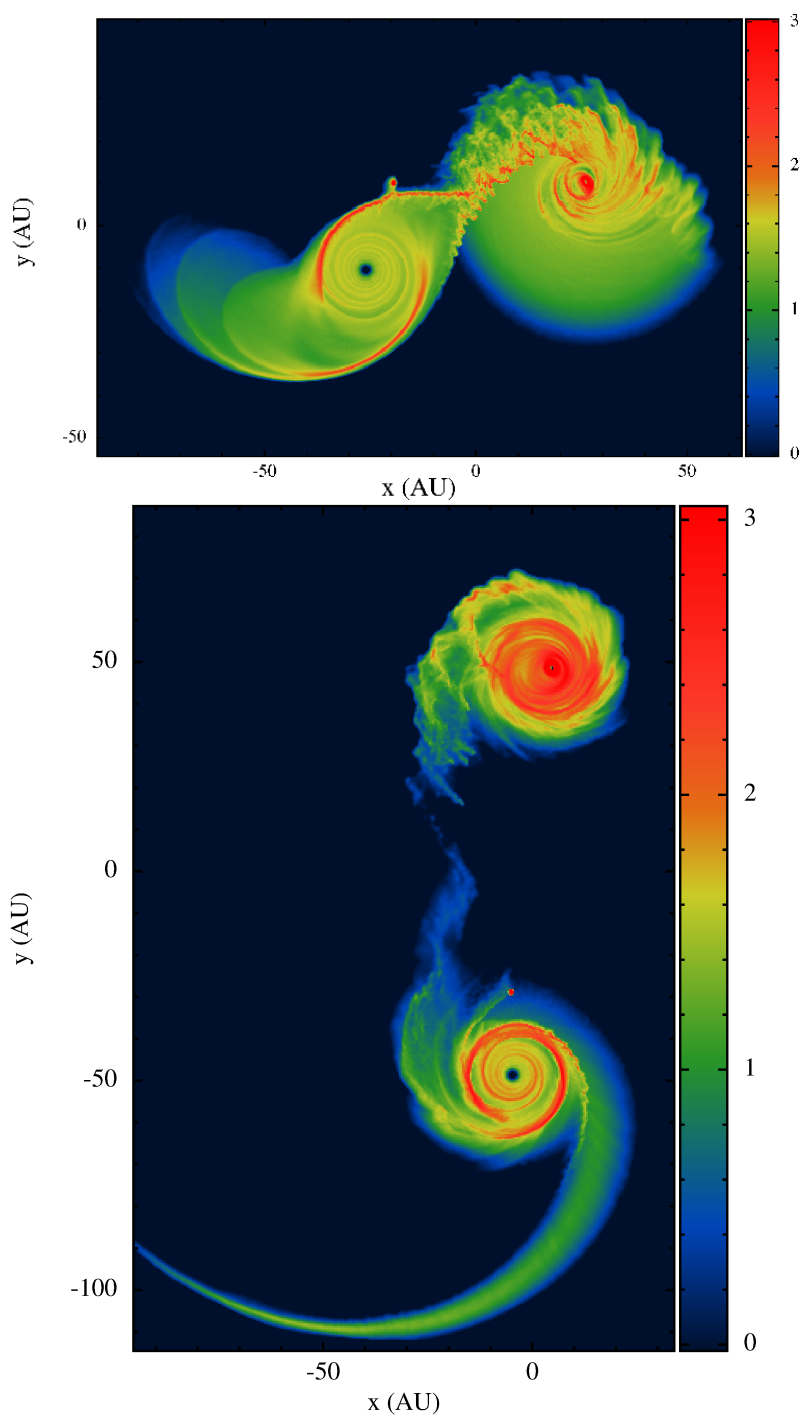

Fig. 10. Logarithm of the superficial density (column integrated) of the circumstellar disks at two different evolutionary steps for the model e. Because of the geometry of the system, the evolution of the two disks is asymmetric.

(Triaud et al. 2010). The inclination evolution due to a series of stellar flybys would appear as a random walk with jumps that may lead to positive or negative changes of the planet (and disk) inclination. The step size of this random walk would also be small, since we consider an extreme case with a very deep flyby. An event like this would be rare in the lifetime of the star within the cluster and its probability would rapidly decrease with time.

We find some significant differences between 2D and 3D simulations.

- The mass lost by the primary disk during the stellar flyby depends on the 3D geometry of the encounter and does not lead to the large mass loss observed in 2D simulations. The difference is mainly due to the inclusion of a disk around the passing star that exchanges mass with the primary disk during the close encounter and the use of an SPH code that allows us to better track the evolution of the gas far away from the stars.

- The planet migrates inward at a slower rate in 3D simulations than in 2D models. The migration of an eccentric orbit,

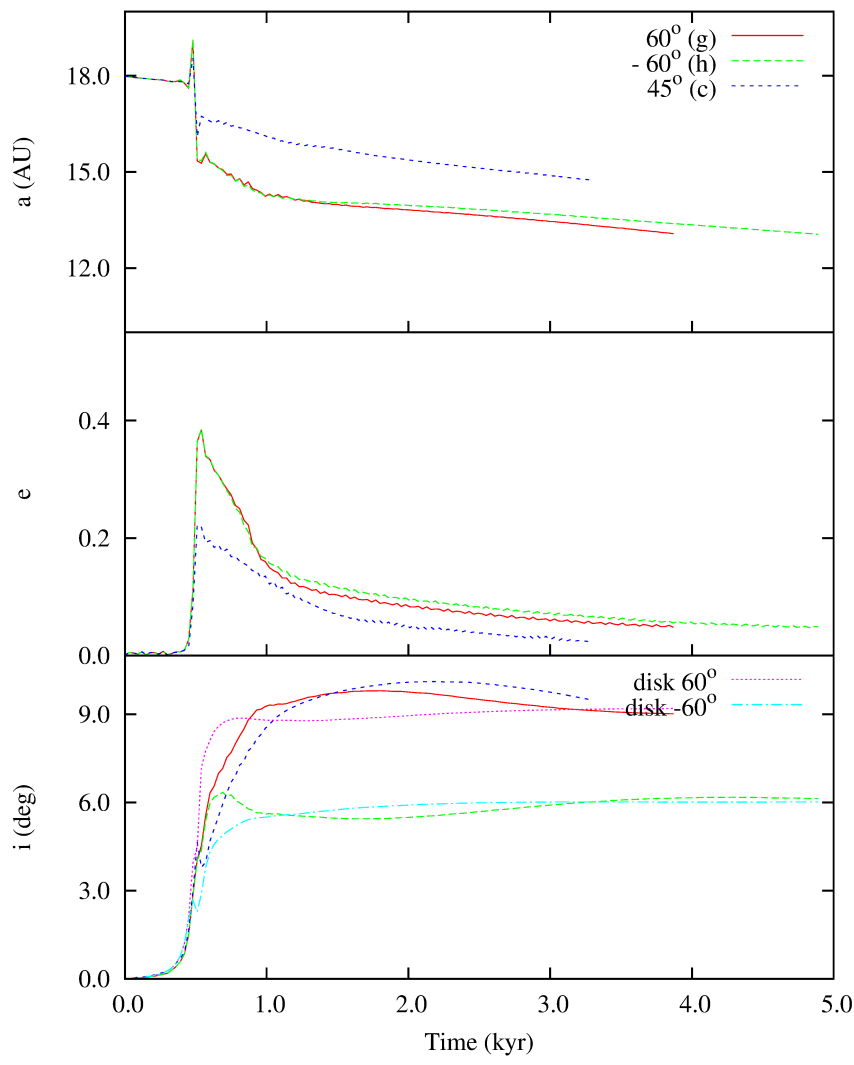

Fig. 11. Evolution of the planet orbital elements $(a, e, i)$ in cases $\mathrm{g}$ and $\mathrm{h}$, compared with the test case c. In the bottom panel we also plot the inclinations of the primary disks in the two models $g$ and $h$ (labeled disk $60^{\circ}$ and disk $-60^{\circ}$ ). The bottom panel shows that the planet is dragged to the disk plane on a short timescale $(\sim 3 \mathrm{Kyr})$.

like that of the planet just after the encounter, appears to be significantly less efficient in 3D models.

- The initial eccentricity excited by the stellar flyby is damped more quickly in 3D models and this is partly due to the reduced amount of mass lost by the disk.

We also observed a slower eccentricity damping efficiency when the orbit of the planet is inclined with respect to the disk due to a slightly weaker disk-planet interaction.

Concerning the effects of stellar flybys on planetary systems in stellar clusters, we do not expect any signatures of the stellar encounters in the eccentricity of the planets. Some inclination between the planet orbit and the star's equatorial plane may appear, but it would be limited. The recent discoveries of strongly misaligned transiting exoplanets (Triaud et al. 2010) must rely on additional dynamical mechanisms (Chatterjee et al. 2008; Nagasawa et al. 2008; Thies et al. 2011; Batygin 2012).

A period of fast Type II migration may occur after the stellar encounter since any gap created by the planet before the flyby is destroyed during the strong interaction between the stars and the disks at the pericenter. A new gap is created when the planet is finally pulled back into the disk midplane and its orbit circularized. The rate at which the disks are destroyed in clusters may be slower than expected since when two stars have an encounter, their disks exchange mass rather than loosing it.

Marzari \& Picogna (2013) explored the consequences of stellar encounters on systems of three planets and a similar exploration would also be interesting in $3 \mathrm{D}$ to test the occurrence of a so-called jumping Jupiter chaotic phase and derive its final 
outcome. The parameter space is extremely large and many different configurations can be envisaged in light of recent discoveries of extrasolar planetary systems. However, according to our results, it appears reasonable to expect that if the damping works in the extreme conditions we have simulated (low disk density, very deep encounter's) it should also be effective in different, potentially more complex, scenarios.

\section{References}

Allen, L., Megeath, S. T., Gutermuth, R., et al. 2007, in Protostars and Planets V (Tucson: University of Arizona Press), 361

Ayliffe, B. A., \& Bate, M. R. 2009, MNRAS, 393, 49

Batygin, K. 2012, Nature, 491, 418

Bell, C. P. M., Naylor, T., Mayne, N. J., Jeffries, R. D., \& Littlefair, S. P. 2013, MNRAS, 434, 806

Bitsch, B., \& Kley, W. 2011, A\&A, 530, A41

Chandar, R., Fall, S. M., \& Whitmore, B. C. 2010, ApJ, 711, 1263

Chatterjee, S., Ford, E. B., Matsumura, S., \& Rasio, F. A. 2008, ApJ, 686, 580

Cresswell, P., Dirksen, G., Kley, W., \& Nelson, R. P. 2007, A\&A, 473, 329

Dukes, D., \& Krumholz, M. R. 2012, ApJ, 754, 56

Fall, S. M., Chandar, R., \& Whitmore, B. C. 2009, ApJ, 704, 453

Forgan, D., \& Rice, K. 2009, MNRAS, 400, 2022
Fragner, M. M., \& Nelson, R. P. 2009, A\&A, 505, 873 Gingold, R. A., \& Monaghan, J. J. 1977, MNRAS, 181, 375

Haisch, Jr., K. E., Lada, E. A., \& Lada, C. J. 2001, ApJ, 553, L153

Hall, S. M., Clarke, C. J., \& Pringle, J. E. 1996, MNRAS, 278, 303 Hillenbrand, L. A. 1997, AJ, 113, 1733

Malmberg, D., \& Davies, M. B. 2009, MNRAS, 394, L26

Malmberg, D., de Angeli, F., Davies, M. B., et al. 2007, MNRAS, 378, 1207

Malmberg, D., Davies, M. B., \& Heggie, D. C. 2011, MNRAS, 411, 859

Marzari, F., \& Nelson, A. F. 2009, ApJ, 705, 1575

Marzari, F., \& Picogna, G. 2013, A\&A, 550, A64

Meru, F., \& Bate, M. R. 2012, MNRAS, 427, 2022

Monaghan, J. J., \& Gingold, R. A. 1983, J. Comput. Phys., 52, 374

Nagasawa, M., Ida, S., \& Bessho, T. 2008, ApJ, 678, 498

Nelson, A. F., Wetzstein, M., \& Naab, T. 2009, ApJS, 184, 326

Palla, F., \& Stahler, S. W. 2000, ApJ, 540, 255

Picogna, G., \& Marzari, F. 2013, A\&A, 556, A148

Price, D. J., \& Monaghan, J. J. 2004, MNRAS, 348, 139

Price, D. J., \& Monaghan, J. J. 2007, MNRAS, 374, 1347

Shakura, N. I., \& Sunyaev, R. A. 1973, A\&A, 24, 337

Springel, V., \& Hernquist, L. 2002, MNRAS, 333, 649

Thies, I., Kroupa, P., Goodwin, S. P., Stamatellos, D., \& Whitworth, A. P. 2011, MNRAS, 417, 1817

Triaud, A. H. M. J., Collier Cameron, A., Queloz, D., et al. 2010, A\&A, 524, A 25

Wetzstein, M., Nelson, A. F., Naab, T., \& Burkert, A. 2009, ApJS, 184, 298

Zakamska, N. L., \& Tremaine, S. 2004, AJ, 128, 869 